ESJ Natural/Life/Medical Sciences

\title{
Evaluation De La Satisfaction Des Patientes Hospitalisées En Suites De Couches À La Maternité De L'hôpital De Bè (Togo)
}

\author{
Ajavon D.R.D.
}

Département de Gynécologie obstétrique, Université de Kara

Kakpovi $K$.

Daketse Y.M.S.

Département de médecine et spécialités, Université de Kara

Ketevi A.A.

Douaguibe B.

Département de Gynécologie obstétrique, Université de Lomé

Logbo-Akey K.E.

Département de Gynécologie obstétrique, Université de Kara

Bassowa A.

Département de Gynécologie obstétrique, Université de Lomé

Aboubakari A.S.

Département de Gynécologie obstétrique, Université de Kara

Doi:10.19044/esj.2021.v17n29p210

Submitted: 05 February 2021

Accepted: 06 July 2021

Published: 31 August 2021
Copyright 2021 Author(s)

Under Creative Commons BY-NC-ND

4.0 OPEN ACCESS

Cite As:

Ajavon D.R.D., Kakpovi K., Daketse Y.M.S., Ketevi A.A., Douaguibe B., Logbo-Akey K.E., Bassowa A. \& Aboubakari A.S.(2021). Evaluation De La Satisfaction Des Patientes Hospitalisées En Suites De Couches À La Maternité De L'hôpital De Bè (Togo). European Scientific Journal, ESJ, 17(29), 210.

https://doi.org/10.19044/esj.2021.v17n29p210

\section{Résumé}

Introduction: L'évaluation de la satisfaction montre l'adéquation entre les attentes des patients et la qualité des soins reçus.

Objectif: évaluer la satisfaction des accouchées hospitalisées en suites de couches à la maternité de l'hôpital de Bè.

Patientes et méthode: Il s'est agi d'une étude transversale à visée descriptive et analytique menée du 1er juillet au 31 août 2018 soit une durée de 2 mois. Elle a eu pour cadre, le secteur des suites de couches de la maternité de l'hôpital de Bè. La collecte des données par interview directe a été faite à l'aide d'une fiche d'enquête anonyme sur le modèle Saphora-MCO 
questionnaires Project COMPAQH et adapté à nos réalités. Le traitement et l'analyse des données ont été réalisées grâce aux logiciels Epi data et Epi info.

Résultats: Au total 300 accouchées ont accepté participer à l'étude. L'âge moyen était de $29 \pm 6,2$ ans. La satisfaction globale était de 94\%. Les principaux éléments de satisfaction étaient : l'accueil (94,7\%), le respect de l'intimité (96,7\%), la prise en charge des soins (94\%), le délai d'attente (86,7\%). L'insatisfaction était due à l'insuffisance de communication des soignants (65,7\%) et de propreté des chambres et des sanitaires (57\%). L’âge, le niveau d'instruction, la parité, le mode d'accouchement avaient influencé significativement l'insatisfaction des patientes.

Conclusion: Cette étude a permis de relever les éléments d'insatisfaction des clientes. Il est opportun d'associer les clients dans le processus de la qualité des soins à l’hôpital de Bè.

Mots clés: Satisfaction, Qualité Des Soins, Accouchées, Togo

\title{
Assessment Of The Satisfaction Of Patients Hospitalized At Maternity Of Bè Hospital (Togo)
}

Ajavon D.R.D.

Département de Gynécologie obstétrique, Université de Kara Kakpovi K. Daketse Y.M.S.

Département de médecine et spécialités, Université de Kara Ketevi A.A. Douaguibe $B$.

Département de Gynécologie obstétrique, Université de Lomé Logbo-Akey K.E.

Département de Gynécologie obstétrique, Université de Kara Bassowa A.

Département de Gynécologie obstétrique, Université de Lomé Aboubakari A.S.

Département de Gynécologie obstétrique, Université de Kara

\begin{abstract}
Introduction: Satisfaction assessment shows the match between patient expectations and the quality of care received.

Objective: to assess the satisfaction of women hospitalized in the maternity ward of the obstetrics and gynecology department of the Bè hospital in order to contribute to the improvement of the quality of care in the maternity ward.
\end{abstract}


Patients and method: This was a cross-sectional study with descriptive and analytical aims conducted from July 1 to August 31, 2018, i.e., a duration of 2 months. It took place in the puerperium section at the maternity of the Bè hospital. Data collection by direct interview was done using an anonymous survey form on the Saphora-MCO questionnaires Project COMPAQH model and adapted to our realities. Data processing and analysis were carried out using Epi data and Epi info software.

Results: A total of 300 women agreed to participate in the study. The average age was $29 \pm 6.2$ years. Overall satisfaction was $94 \%$. The main elements of satisfaction were: reception (94.7\%), respect for privacy (96.7\%), management of care (94\%), waiting time (86.7\%). Dissatisfaction was due to insufficient communication from caregivers (65.7\%) and cleanliness of rooms and toilets (57\%). Age, level of education, parity and mode of delivery had a significant influence on the respondent's dissatisfaction.

Conclusion: This study identified factors that led to patient's dissatisfaction. It is appropriate to involve patients in the quality of care process at the Bè hospital.

Keywords: Satisfaction, Quality Of Care, Deliveries, Togo

\section{Introduction}

La satisfaction est l'écart entre deux notions évolutives : la qualité perçue et la qualité attendue. La satisfaction des patientes est donc un indicateur de la qualité des soins. La qualité des soins tient compte de l'expérience des femmes, des nouveau- nés et de leurs familles (OMS 2017). La satisfaction du patient est ainsi le reflet des préférences personnelles du patient, de ses attentes, et de la réalité de la prise en charge (OMS 2017, Kotler $P$, 2005). Selon le plan national de développement sanitaire du Togo, la réduction de la mortalité maternelle passe par les soins obstétricaux de qualité pour l'atteinte des objectifs du développement durable (PNDS 2017).

L'évaluation de la satisfaction des patients est influencée par plusieurs facteurs. Selon certaines études, l'information reçue et la communication avec les soignants sont les facteurs de satisfaction les plus importants (Detlef Saal T et al. 2006 ; Collet 2008 ; Bougzima I et al 2011). D’autres études ont souligné une prise en charge adaptée et efficace de la douleur ainsi que l'assurance d'un bon confort (Detlef Saal T et al. 2006 ; Adamou H et al. 2017). La satisfaction des patients serait fonction du contexte culturel (Beninguisse G et al. 2004 ; Mendoza Aldana J et al. 2001 ; Bernitz S 2016). Peu d'études sur la satisfaction des patientes ont été réalisées au Togo, pourtant ce type de travail contribue à l'amélioration de la qualité des soins (OMS 2017, Adamou H. et al. 2017). L'objectif de cette étude était d'évaluer 
la satisfaction des accouchées en suites de couches à la maternité de l'hôpital de Bè.

\section{Patientes et méthodes}

Il s'est agi d'une étude transversale à visée descriptive et analytique réalisée du $1^{\mathrm{er}}$ juillet au 31 août 2018 soit une durée de 2 mois. Notre étude s'est déroulée dans le secteur des suites de couches de la maternité de l'Hôpital de Bè au Togo. L'hôpital de Bè compte 112 lits avec un taux d'occupation de $44 \%$ en 2016. Trois sages-femmes, une accoucheuse auxiliaire et deux accoucheuses permanentes se relayent de jour comme de nuit pour le suivi des femmes hospitalisées en suites de couches (Données SMI 2017).

Etaient incluses les accouchées hospitalisées dans le secteur des suites de couches de la maternité, quel que soit l'issue de la grossesse, ayant bénéficié d'un exéat pendant la période d'étude.

La collecte des données a été faite à l'aide d'une fiche d'enquête anonyme comportant 27 questionnaires et 6 déterminants. La fiche a été réalisée sur le modèle Saphora-MCO questionnaires Project COMPAQH et adaptée à nos réalités. Le consentement oral de chaque accouchée ou de ses parents était obtenu avant l'administration des questionnaires par entretien direct. Pour les patientes qui ne comprenaient pas français, les questions ont été traduits en mina (langue vernaculaire parlé au Togo) par l'enquêteur.

Les paramètres étudiés étaient les caractéristiques socio démographiques (âge, statut marital, niveau d'instruction, provenance, statut de la grossesse), les antécédents obstétricaux (parité, mode d'accouchement et issue de la grossesse), l'accueil des patientes et/ou leurs accompagnateurs, le délai d'attente avant consultation, le respect de l'intimité et de la dignité, les informations reçues par la patiente (identification du prestataire, écoute du personnel, obtention d'une information claire sur le déroulement des examens cliniques et paracliniques et sur l'organisation sur la sortie de la patiente), les prestations des soins (soins médicaux et paramédicaux et prise en charge de la douleur) et les conditions de séjour (confort des chambres, propreté des chambres et des sanitaires).

Les différentes réponses obtenues auprès des enquêtés ont permis de classer le niveau de satisfaction en trois catégories : très satisfait, satisfait, pas satisfait.

Le traitement et l'analyse des données ont été réalisés grâce aux logiciels Epi data version 3.1 et Epi Info 2000 version 3.5.6. Le test statistique de Khi-deux de Pearson avec $\mathrm{p}<0,05$ (valeur de signifiance) était utilisé pour faire les liens entre les variables et le niveau de satisfaction. 


\section{Résultats}

\section{Caractéristiques socio démographiques}

Du $1^{\text {er }}$ juillet au 31 août 2018, sur 351 accouchées en suites de couches, 300 ont accepté participer à l'enquête soit un taux de réponse de 85,5\%. L'âge moyen des accouchées était de $29 \pm 6,2$ ans (extrêmes de 16 et 49 ans). La figure 1 donne la répartition par tranche d'âge. Aussi 84,7\% (254) provenaient de Lomé et 15,3\% (46) hors de Lomé. Pour le niveau d'instruction : 53,3\% $(n=160)$ avaient un niveau secondaire; $22 \%(n=66)$ niveau primaire, $13 \%$ $(n=39)$ non instruites et $11,7 \%(n=35)$ niveau supérieur. Plus de $86 \%(n=259)$ étaient mariées et $13,7 \%(n=41)$ célibataires. Les grossesses étaient désirées dans 67,7\% ( $n=203)$ et non désirées dans 32,3\% ( $n=97)$.

Figure 1. Répartition des patientes selon l'âge

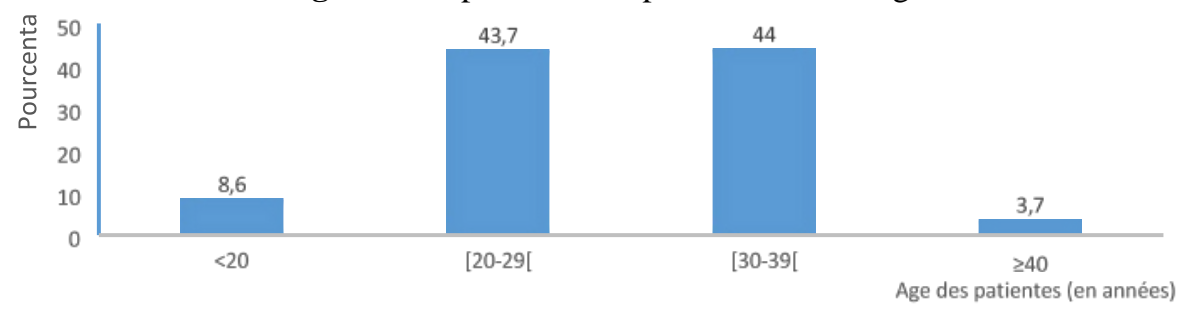

\section{Antécédents obstétricaux}

Parité : les multipares représentaient $66,7 \%(n=200)$ et les primipares $33,3 \%(n=100)$.

Mode d'accouchement : l'accouchement était réalisé par voie basse eutocique dans $41 \%(n=125)$, instrumental dans $1 \%(n=2)$. La césarienne d'urgence représentait 33\% ( $n=99)$ et la césarienne programmée $25 \%(n=74)$.

Issue de la grossesse : les nouveaux nés étaient vivants dans 97,3\% $(\mathrm{n}=292)$ et mort-nés ou décédés à la naissance dans 2,7\% $(\mathrm{n}=8)$ des cas.

\section{Satisfaction globale}

A l'issue de leur séjour, 94\% des accouchées étaient satisfaites dont $4 \%(n=12)$ étaient très satisfaites et $90 \%(n=270)$ étaient satisfaites. Par contre $6 \%(n=18)$ n'étaient pas satisfaites. Parmi les accouchées satisfaites 96,7\% ( $\mathrm{n}=290)$ avaient affirmé qu'elles pourraient revenir à l'hôpital de Bè pour les mêmes motifs.

\section{Satisfaction spécifique des accouchées hospitalisées Accueil des patientes et de leurs familles}

Notre échantillon révélait que 94,7\% ( $\mathrm{n}=284)$ étaient satisfaites de l'accueil. Aussi 95,7\% (n=287) patientes hospitalisées ont apprécié l'attitude du personnel envers leurs parents lors de leur visite durant leur séjour en hospitalisation, voir tableau I. 


\section{Délai d'attente}

Quatre-vingt-six virgule sept pour cent $(\mathrm{n}=260)$ étaient satisfaites du délai d'attente inférieur à 30 minutes avant la consultation, voir tableau I.

\section{Respect de l'intimité et dignité des patientes}

Dans notre échantillon, 96,7\% $(n=290)$ des patientes étaient satisfaites des précautions prises pour respecter leur intimité et leur dignité tout au long de leur séjour, voir tableau I.

\section{Informations reçues par la patiente sur son état}

Identification du prestataire : plus de 34\% (n=104) des accouchées ne connaissaient pas le profil du personnel ayant assisté à leur accouchement tandis que 196 (65,3\%) ont cité les médecins, assistants médicaux, sagesfemmes, accoucheuses comme les membres du personnel soignant ayant assisté à 'accouchement.

Concernant les informations données sur le déroulement des examens para cliniques et le traitement, $90 \%(n=270)$ des accouchées étaient satisfaites Écoute des plaintes: Sur les 91 accouchées qui avaient des plaintes, 82 patientes hospitalisées soit $90 \%$ avaient déclaré que leurs plaintes ont été écoutées par le personnel.

Obtention de réponses aux questions : la plupart des accouchées n'avait pas posé des questions au personnel $(73,3 \%, n=220)$. Sur les 80 qui avaient posé des questions, $7,5 \%(\mathrm{n}=6)$ étaient très satisfaites des réponses et $17,5 \%(n=14)$ satisfaites, voir tableau I.

\section{Soins médicaux et paramédicaux}

La qualité de la prise en charge par les équipes médicales et paramédicales a été satisfaisante selon $94 \%$ ( $\mathrm{n}=282)$ accouchées. Aussi la prise en charge de la douleur était satisfaisante chez $86,3 \%(n=259)$ des patientes hospitalisées, voir tableau I.

\section{Conditions du séjour}

La durée moyenne d'hospitalisation des accouchées était de 4,5 $\pm 2,4$ jours avec des extrêmes de 2 jours et 15 jours. Le séjour est d'autant plus long que l'accouchement a été fait par césarienne d'urgence. Dans notre échantillon, 259 accouchées soit 63,3\% étaient satisfaites du confort de la chambre (mobilier, calme, température, télévision, moustiquaire). Cependant 171 accouchées (57\%) n'étaient pas satisfaites de la propreté des chambres et des sanitaires lors de leur séjour en hospitalisation, voir tableau I. 


\section{Organisation sur la sortie de la patiente}

Elle a concerné l'obtention d'une information sur les symptômes d'alerte, le suivi des consultations post natales et le coût d'hospitalisation. L’organisation de la sortie de l'hôpital était satisfaisante selon 94\% (n=282) des patientes hospitalisées. Le coût d'hospitalisation par jour est fixé à 1500 francs CFA et la durée moyenne était de 4,5jours ; soit le coût moyen d'hospitalisation était de 6750 francs CFA. Près de $96 \%(n=287)$ des patientes étaient satisfaites du coût de l'hospitalisation, voir tableau I.

Tableau I. Répartition des accouchées selon les éléments de satisfaction

\begin{tabular}{|c|c|c|c|c|}
\hline & $\begin{array}{c}\text { Très } \\
\text { satisfait }\end{array}$ & Satisfait & $\begin{array}{c}\text { Pas } \\
\text { satisfait }\end{array}$ & Total \\
\hline & $\mathbf{n ~ ( \% )}$ & $\mathbf{n ~ ( \% )}$ & $\mathbf{n}(\mathbf{\%})$ & \\
\hline Accueil & $108(36 \%)$ & $\mathbf{1 7 6}(\mathbf{5 8 , 7} \%)$ & $16(5,3 \%)$ & 300 \\
\hline Délai d'attente & $\mathbf{1 8 6}(\mathbf{6 2} \%)$ & $74(24,7 \%)$ & $40(13,5 \%)$ & 300 \\
\hline Soins & $121(40,3 \%)$ & $161(53,7 \%)$ & $18(6 \%)$ & 300 \\
\hline Prise en charge douleur & $87(29 \%)$ & $172(57,3 \%)$ & $41(13,7 \%)$ & 300 \\
\hline Respect intimité & $99(33 \%)$ & $\mathbf{1 9 1}(\mathbf{6 3 , 7 \% )}$ & $10(3,3 \%)$ & 300 \\
\hline Confort des chambres & $19(6,3 \%)$ & $171(57 \%)$ & $110(36,7 \%)$ & 300 \\
\hline *Propreté chambres & $13(4,3 \%)$ & $119(39,7 \%)$ & $\mathbf{1 6 8}(\mathbf{5 6 \% )}$ & 300 \\
\hline Organisation sortie & $28(9,3 \%)$ & $\mathbf{2 5 4}(\mathbf{8 4 , 7 \% )}$ & $18(6 \%)$ & 300 \\
\hline Information sur les soins & $161(53,7 \%)$ & $109(36,3 \%)$ & $30(10 \%)$ & 300 \\
\hline Réponses aux questions & $06(7,5 \%)$ & $14(17,5 \%)$ & $\mathbf{6 0} \mathbf{( 7 5 \% )}$ & 80 \\
\hline Ecoute des plaintes & 00 & $82(27,3)$ & $9(3 \%)$ & 91 \\
\hline
\end{tabular}

*propreté des chambres et sanitaires

\section{Raisons d'insatisfaction}

Les principales raisons d'insatisfaction étaient la relation avec les soignants : identification du prestataire $(65,3 \%)$, réponses aux questions (75\%), plaintes (3\%) et les conditions de séjour : propreté des chambres et sanitaires (57\%), confort des chambres (32,7\%).

\section{Facteurs influençant la satisfaction des accouchées hospitalisées}

L’âge, le niveau d'instruction, la parité et le mode d'accouchement, le statut ainsi que l'issue de la grossesse ont influencé significativement la satisfaction des accouchées hospitalisées. (Tableau II). 
Tableau II. Répartition des facteurs influençant la satisfaction des accouchées hospitalisées sur les conditions de séjour (confort des chambres, propreté des chambres/sanitaires)

\begin{tabular}{|c|c|c|c|}
\hline & Satisfait (n) & Pas satisfait (n) & $\mathbf{p}$ \\
\hline $\begin{array}{c}\text { Confort des chambres } \\
\text { Age (ans) } \\
<20(\mathrm{n}=26) \\
20-29(\mathrm{n}=131) \\
30-39(\mathrm{n}=132) \\
\geq 40(\mathrm{n}=11)\end{array}$ & $\begin{array}{c}(\mathrm{n}=190) \\
\\
23 \\
88 \\
73 \\
6\end{array}$ & $\begin{array}{c}(\mathrm{n}=110) \\
3 \\
43 \\
59 \\
5\end{array}$ & 0.007 \\
\hline $\begin{array}{l}\text { Niveau d'instruction } \\
\text { Non instruite }(n=39) \\
\text { Primaire }(n=66) \\
\text { Secondaire }(n=160) \\
\text { Supérieur }(n=35)\end{array}$ & $\begin{array}{c}31 \\
48 \\
102 \\
9\end{array}$ & $\begin{array}{r}8 \\
18 \\
58 \\
26\end{array}$ & 0.0000 \\
\hline $\begin{array}{c}\text { Mode d'accouchement } \\
\text { Normal }(\mathrm{n}=125) \\
\text { Urgence }(\mathrm{n}=99) \\
\text { Programmée }(\mathrm{n}=74) \\
\text { Instrumental }(\mathrm{n}=2) \\
\end{array}$ & $\begin{array}{c}91 \\
63 \\
36 \\
0\end{array}$ & $\begin{array}{r}34 \\
36 \\
38 \\
2\end{array}$ & 0.001 \\
\hline $\begin{array}{c}\text { Statut de grossesse } \\
\text { Desirée }(\mathrm{n}=203) \\
\text { Non désisrée }(\mathrm{n}=97) \\
\text { Issue de la grossesse } \\
\text { *Nné vivant }(\mathrm{n}=292) \\
\text { *Nné décédé }(\mathrm{n}=8) \\
\text { Propreté des chambres/ } \\
\frac{\text { sanitaires }}{\text { Parité }} \\
\text { Primipares }(\mathrm{n}=100) \\
\text { Multipares }(\mathrm{n}=200) \\
\text { Niveau d'instruction } \\
\text { Non instruite } \mathrm{n}=39 \\
\text { Primaire } \mathrm{n}=66 \\
\text { Secondaire } \mathrm{n}=160 \\
\text { Supérieur } \mathrm{n}=35\end{array}$ & $\begin{array}{c}119 \\
71 \\
182 \\
8 \\
\\
(n=132)\end{array}$ & $\begin{array}{rr}84 & 01 \\
26 & \\
182 & 0,02 \\
0 & \\
& \\
& \\
(\mathrm{n}=168) & \end{array}$ & $\begin{array}{l}0,004 \\
0,004\end{array}$ \\
\hline
\end{tabular}

P: valeur de signifiance $(<0,05) \quad$ Nné: nouveau-né $\quad$ n: effectif

\section{Discussion}

La majorité des enquêtées (94\%) était satisfaite de leur séjour à l’hôpital de Bè. D’autres études ont retrouvé des taux élevés de satisfaction : Collet, France 2006 (95\%) ; Doumbia, Côte d'ivoire 2016 (93,1\%) ; Melese, Ethiopie 2014 (85,4\%) (Collet M. 2008, Doumbia M et al. 2016, Melese T et al. 2014) tandis que Bougmiza, Tunisie 2011, avait retrouvé un taux plus faible de 51\% (Bougmiza I et al. 2011). L’appréciation de la qualité des soins 
suppose une connaissance préalable des standards de prise en charge (OMS 2017, Mategic B et al. 2014).

L'âge, le niveau d'instruction, la parité et le mode d'accouchement avaient influencé significativement la satisfaction des accouchées hospitalisées. Ce constat a été fait par d'autres auteurs (Bougmiza I et al. 2011, Melese T et al. 2014, Traoré SO et al. 2019). Ainsi le taux de satisfaction décroit quand le niveau d'instruction s'améliore donc les non instruites étaient plus satisfaites.

La plupart des accouchées hospitalisées (94,7\%) et leurs parents (95,7\%) étaient satisfaits de l'accueil. Le délai d'attente avant la consultation a été jugé satisfaisante (86,7\%). Notre résultat se rapproche de celui de Melese (Melese $\mathrm{T}$ et al. 2014) qui avait trouvé 88,2\%.

Le respect de la dignité fait référence au standard 5 de la qualité des soins de l'OMS (OMS 2017). Dans notre étude, 96,7\% des patientes étaient satisfaites des précautions prises pour garantir leur intimité et leur dignité tout au long de leur séjour. Nos résultats sont largement au-dessus de ceux de Melese et al (Melese T et al. 2014) qui avaient trouvé 78,9\% de satisfaction du fait d'une insuffisance dans le respect des politiques et stratégies de soins aux patients dans le système de santé.

Parmi les patientes hospitalisées, deux cent quatre-vingt-deux (94\%) étaient satisfaites par rapport à la qualité de la prise en charge médicale et paramédicale. La prise en charge de la douleur est un élément essentiel de la satisfaction des soins (Detlef Saal T et al.2006, Melese T et al. 2014, OMS 2018). Notre étude révélait que $86,3 \%$ des patientes étaient satisfaites de la prise en charge de la douleur tandis que $13,4 \%$ ne l'étaient pas. Cette insatisfaction pourrait s'expliquer par le fait que cette expérience est vécue seule par les patientes qui ne sont pas soutenues par leurs accompagnants dans la salle de travail où l'accès est interdit à toute personne autre que le personnel soignant. Selon l'OMS, la présence des accompagnants est indispensable pour une expérience positive des soins intrapartum (OMS 2018).

L'insatisfaction des accouchées était liée principalement à l'insuffisance de communication et les conditions de séjour.

Peu d'accouchées étaient satisfait des conditions de séjour lors de l'hospitalisation. Les accouchées satisfaites de la propreté des chambres et des sanitaires étaient primipares, avaient un âge compris entre 20-29ans, un niveau d'instruction primaire et accouché par césarienne en urgence. Mategic en Serbie avait noté que les mères jeunes et les multipares étaient moins préoccupées par les conditions environnementales (Mategic B et al. 2014). D'autre part, les accouchées insatisfaites des conditions de séjour avaient accouché par une césarienne de convenance. Aussi, la durée de séjour était plus longue en cas d'accouchement par césarienne. Il existe un lien significatif entre le confort de la chambre et l'issue de la grossesse. Selon l'étude de 
Dombia en Côte d'ivoire, les insatisfactions liées aux conditions de séjour ont porté généralement sur la commodité des suites de couches (la propreté de la salle, l'étroitesse des lits et la propreté des couvertures), le niveau de bruit et la température (chaleur excessive) (Doumbia M et al. 2016).

La satisfaction des femmes à l'égard des soins prénatals, intrapartum et postnatals était associée à une plus grande empathie (Bougmiza I et al. 2011, Mendoza Aldana J et al. 2001, Bazant E S et al. 2009, Tesfaye R et al. 2016, Okonofua F et al. 2017, Mehata S et al. 2017). La communication avec les femmes et leur famille répond aux besoins et aux préférences des intéressés. Une communication efficace entre les soignants et les patientes permet d'éviter l'anxiété (OMS 2017). Selon notre étude, 90\% des patientes étaient satisfaites des informations données sur le déroulement des examens para cliniques et le traitement. Bougzima avait également retrouvé que l'évaluation des soins médicaux et paramédicaux était généralement bonne (Bougmiza I et al. 2011). Par contre, 65,3\% ne connaissaient pas l'identité du prestataire de soins. Aussi $75 \%$ n'ont pas eu de réponses à leurs questions et 3\% n’ont pas eu de satisfaction à leurs plaintes. Melese avait trouvé que ce faible taux de satisfaction était dû à la surcharge de travail des prestataires. La mauvaise communication soignant-soigné est un facteur incriminé dans l'insatisfaction (Adamou H et al. 2017). Dans l'étude de Collet en France, une femme sur dix juge les informations fournies insuffisantes et une sur vingt inexistantes en cas de césarienne (programmée ou non). L'insuffisance de la communication est à l'origine de la plupart des litiges voire des conflits entre soignants et soignés. Il est urgent de veiller à la formation de tous les acteurs de santé et en leur permettant de consacrer l'essentiel de leur temps aux malades. La communication est gage d'une qualité essentielle de la relation soignantsoigné (Okonofua F et al. 2017, Mantz JM 2006).

Avoir l'occasion de poser des questions était positivement associé à la satisfaction des clients (Mehata $S$ et al. 2017). Dans notre étude, 73,3\% n'avaient pas posé de question. La majorité des patientes hospitalisées (69,7\%) n'avaient pas d'opinion. Cela démontre une fois de plus l'insuffisance dans la relation d'aide. La satisfaction des patients serait fonction du contexte culturel (Beninguisse G et al. 2001, Mendoza Aldana J et al. 2001). L'étude de Jorge avait retrouvé que les soins qui satisfont tous les besoins médicaux ne répondent pas forcément aux besoins affectifs et sociaux des patients et que l'on se trouve donc devant un dilemme. Il suggère que les pays en développement qui privilégient les services de santé axés sur les malades entreprennent des recherches plus approfondies sur les déterminants de la satisfaction des patients dans leurs cultures respectives (Mendoza Aldana $\mathrm{J}$ et al. 2001). Il importe de placer la responsabilité de donner des soins de qualité au cœur de la relation de soins patient-soignant (Mehata S et al. 2017). 
La plupart des patientes (95,7\%) étaient satisfaites du coût de leur séjour. Le coût élevé des prestations constitue un facteur d'insatisfaction (Tesfaye R et al. 2016, Okonofua F et al. 2017, Mehata S et al. 2017, Hurissa BF et al. 2017). Cependant la qualité des services dans le secteur privé de la santé qui ne reçoit pas de subvention est satisfaisante à cause des commodités et de la prise en charge (Andaleeb SS, 2000). Les aspects interpersonnels des soins et l'éducation sont des éléments importants de la satisfaction maternelle (Mategic B et al. 2014, Bazant E S et al. 2009). L'amélioration de la qualité passe donc nécessairement par une adéquation de l'offre aux attentes et préférences et fait appel à une complémentarité dans l'action entre les systèmes traditionnel et biomédical pour la définition d'un paquet minimum consensuel de soins obstétricaux qui garantit à la fois l'efficacité clinique et la satisfaction des clients (Beninguisse $\mathrm{G}$ et al. 2001). Une formation initiale ou continue du personnel sur la communication hospitalière permettrait d'améliorer les soins de maternité (Okonofua F et al. 2017, Mantz JM 2006).

\section{Conclusion}

Cette étude permet de dire que les accouchées, dans leur majorité, étaient satisfaites au cours de leur hospitalisation en suites de couches. Cependant un tiers d'entre elles n'était pas satisfait de la prise en charge médicale et paramédicale mais aussi de la communication avec le personnel et des conditions de séjour. La satisfaction des clientes est corrélée à plusieurs facteurs. L'évaluation de la satisfaction devrait avoir un impact sur la prise en charge globale des accouchées. Il serait important d'associer les clients pour trouver des interventions adaptées à leurs besoins. Ceci permettra de réduire leur insatisfaction en vue d'améliorer la qualité des soins en suites de couches à l’hôpital de Bè.

\section{References:}

1. Organisation Mondiale de la Santé (2017). Standards pour l'amélioration de la qualité des soins maternels et néonatals dans les établissements de santé. Genève : Organisation Mondiale de la Santé, p 2-6

2. Kotler P., Dubois B. (2005) «Satisfaire et fidéliser le client ». In Marketing Management. Paris, France: Pearson; p 68

3. Ministère de la Santé et de la Protection sociale (2017). Plan National de Développement Sanitaire PNDS 2017-2022, République Togolaise, pp 23-26

4. Detlef Saal T., Heidegger M., Nuebling M. (2006). L'évaluation de la satisfaction a-t-elle un sens ? Le praticien en anesthésie réanimation, 10(4) : 305-310. 
5. Collet M. (2008). Satisfaction des usagères des maternités à l'égard du suivi de la grossesse et du déroulement de l'accouchement. Direction de la recherche, des études, de l'évaluation et des statistiques (DRESS), Études et Résultats, $\mathrm{N}^{\circ}$ 660, 6pages. République Française.

6. Bougmiza I., EL Ghardallou M., Zedini C., Lahouimel H., Nabli-Ajmi T., Gataa R., Touati I., Khairi H., Mtiraoui A. (2011). Evaluation de la satisfaction des patientes hospitalisées au service de gynécologie obstétrique de Sousse, Tunisie. Pan African Medical Journal, 8:44 49

7. Adamou H., Adamou Maigagi I., Habou O., Halidou M., Karimou S., Sani R., Abarchi H. (2017). Satisfaction des patients, élément important dans la globalité des soins : cas d'un service des urgences chirurgicales au Niger. Mali medical Tome XXXII,32(2):20-26

8. Beninguisse G., Nikièma B., Fournier P., Haddad S. (2004). L'accessibilité culturelle : une exigence de la qualité des services et soins obstétricaux en Afrique. African Population Studies Sup, 19(2) : 251-264

9. Mendoza Aldana J., Piechulek H., et Al-Sabir A. (2001). Satisfaction des patients et qualité des soins dans les zones rurales du Bangladesh. Bulletin de l'organisation mondiale de la Santé : recueil d'articles, 5 : 65-70

10. Bernitz S., Oian P., Sandvik L., Blix E. (2016). Evaluation of satisfaction with care in a midwifery unit and an obstetric unit: a randomized controlled trial of low risk women. BMC Pregnancy Childbirth: 16: 143

11. District sanitaire N³ (2017). Rapport annuel d'activités. Données SMI-PF DISH 2, République du Togo

12. Projet COMPAQH (2006). Questionnaire (C) Saphora-MCO modifié (C). Tous droits réservés Page 65pages. Satisfaction du patient hospitalisé (consulté le 07/6/2018). Disponible à partir de : URL: https://fr.ccecqa.asso.frdefault-image.

13. Doumbia M., Andoh Kouakou H., Zeregbe T., Achy A., Seka S., Abhe-Gnangoran E. (2016). Suivi de la grossesse et satisfaction des patientes en suites de couche en côte d'ivoire. Revue ivoir anthropol sociol KASA BYA KASA EDUCI, $\mathrm{N}^{\circ} 33: 63-83$

14. Melese T., Gebrehiwot Y., Bisetegne D., Habte D. (2014). Assessment of client satisfaction in labor and delivery services at a maternity referral hospital in Ethiopia. Pan African Medical Journal, 17 : 76

15. Mategic B., Santric Milicevic M., Vasic V., Djikanovic B. (2014). Maternal satisfaction with organized perinatal care in Serbian public hospital. BMC Pregnancy and Childbirth, 14: 14. 
16. Traoré S.O., Doumbia S., Samaké A., Traoré A., Tall S., Dembélé Y., Traoré O.M., Albachar A., Camara D., Traoré M.A., Coulibaly S., Camara K., Traoré M., Dolo A. (2019). Étude de la satisfaction des soins du post-partum immédiat (SSOPPI) au centre de santé de référence de la commune $\mathrm{V}$ de Bamako. Mali Médical Tome XXXIV, 34(2) : 23-29

17. Organisation Mondiale de la Santé (2018). Recommandations de l'OMS sur les soins intrapartum pour une expérience positive de l'accouchement, 8pages. WHO/RHR/18.12

18. Bazant E.S., Koenig M.A. (2009). Women's satisfaction with delivery care in Nairobi's informal settlements. Int J Qual Health Care, 21: 79 - 86.

19. Tesfaye R., Worku A., Godana W., Lindtjorn B. (2016). Client satisfaction with delivery care service and associated factors in the public health facilities of Gamo Gofa zone, southwest Ethiopia: in a resource limited setting”. Hindawi Publishing Corporation, Obstetrics and Gynecology International :1-8

20. Okonofua F., Ogu R., Aholor K., Okike O., Abdus- Salam R., Gana M. (2017). Qualitative assessment of women's satisfaction with maternal health care in referral hospitals in Nigeria". Reproductive Health,; $14: 44$.

21. Mantz J.M. (2006). Importance de la communication dans la relation soignant-soigné. Bulletin de l'Académie Nationale de Médecine, Sciences Direct, Elsevier, 190(9) :1999-2011.

22. Mehata S., Paudel Y.R., Dariang M., Aryal K.K., Paudel S., Mehta R. (2017). Factors determining satisfaction among facility-based maternity clients in Nepal”. BMC Pregnancy and Childbirth, ; 17 : 319.

23. Vissandjée B. (2011). Communication, qualité des soins. Entre-vues, 2(5): 4pages

24. Hurissa B.F., Getinet T. (2017). Assessment of Provision of Women Friendly Care and Associated Factors among Postnatal Mothers at Three Public Hospitals of Jimma zone, Oromia Region, Ethiopia, 2016. J Biomedical Sc ; 6: 26.

25. Andaleeb S.S. (2000). Service quality in public and private hospitals in urban Bangladesh: a comparative study”. Health policy, 53: 25-37. 